\title{
Klotho suppresses tumor progression via inhibiting IGF-1R signaling in T-cell lymphoma
}

\author{
XIANGXIANG ZHOU ${ }^{1}$, YA ZHANG ${ }^{1}$, YING $\mathrm{LI}^{1}$, YANGYANG XU ${ }^{1}$, \\ LINGYAN ZHANG ${ }^{1}$, YING LI $^{1}$ and XIN WANG ${ }^{1,2}$ \\ ${ }^{1}$ Department of Hematology, Shandong Provincial Hospital Affiliated to Shandong University, Jinan, \\ Shandong 250021; ${ }^{2}$ Shandong University School of Medicine, Jinan, Shandong 250012, P.R. China
}

Received November 11, 2016; Accepted April 27, 2017

DOI: $10.3892 /$ or.2017.5744

\begin{abstract}
Klotho is a transmembrane protein and acts as an upstream modulator of insulin-like growth factor-1 receptor (IGF-1R) signaling, which was indicated to be involved in the pathogenesis of solid cancer and hematological malignancies, including T-cell lymphoma. Although Klotho was recently identified as a tumor suppressor in several types of human malignancies, the potential role of Klotho in T-cell lymphoma has not been reported. In the present study, we investigated the expression level and the molecular events of Klotho in T-cell lymphoma. Significantly lower expression of Klotho was observed in T-cell lymphoma patient samples compared to normal lymph nodes. Functional analysis after Klotho overexpression revealed significantly inhibited tumor cell viability in T-cell lymphoma. Moreover, apoptosis of T-cell lymphoma cells were induced after transfected with Klothooverexpressing vectors. Forced expression of Klotho resulted in decline of activation of IGF-1R signaling, accompanied by decreased phosphorylation of its downstream targets, including AKT and ERK1/2. These data indicated that Klotho acts as a tumor suppressor via inhibiting IGF-1R signaling, thus suppressing the viability and promoting apoptosis in T-cell lymphoma. Taken together, Klotho may serve as a potential target for the therapeutic intervention of T-cell lymphoma.
\end{abstract}

\section{Introduction}

T-cell lymphoma is the main aggressive malignancy of T lymphocytes, which is characterized by highly heterogeneous molecular and clinical characteristics. This type of lymphoma

Correspondence to: Professor Xin Wang or Professor Ying Li, Department of Hematology, Shandong Provincial Hospital Affiliated to Shandong University, 324 Jingwu Road, Jinan, Shandong 250021, P.R. China

E-mail: xinw007@126.com

E-mail: liyjn@126.com

Key words: T-cell lymphoma, Klotho, IGF-1R, proliferation, apoptosis is generally resistant to chemotherapy (1). Identification of the molecular abnormalities involved in the development of T-cell lymphoma will improve the classification and provide novel therapeutic strategy.

Klotho was considered an anti-aging gene when it was originally identified (2). Klotho homozygous mutant mice $\left(\mathrm{kl}^{-/}\right)$ developed multiple premature aging syndromes, including hypogonadism, skin atrophy, and pulmonary emphysema $(2,3)$. Klotho gene is located in chromosome 13q12 in human with the size of $50 \mathrm{~kb}$ (2). Klotho protein consists of an extracellular domain, a single transmembrane domain and an intracellular domain. The extracellular domain (secreted Klotho), consisting of KL1 and KL2, could be released into the serum to function as a circulating hormone to regulate the activity of oxidative stress and multiple growth factor receptors $(4,5)$.

Accumulating evidence has implicated that Klotho is extensively downregulated in several solid malignancies (6-9). Klotho was revealed to modulate the activity of several signaling pathways, including the FGF signaling, insulin-like growth factor-1 receptor (IGF-1R) and Wnt pathways (5,10-12). These pathways also play crucial roles in the development of T-cell lymphoma $(13,14)$. However, the role of Klotho in T-cell lymphoma has not been reported.

In the present study, we hypothesized that Klotho was aberrantly expressed and involved in the pathogenesis in T-cell lymphoma. We observed decreased expression of Klotho in the patient tissues and cell lines of T-cell lymphoma. The anti-proliferative and pro-apoptotic effect of Klotho were further identified in T-cell lymphoma cells. Klotho could act as an upstream regulator of IGF-1R signaling in this disease. The data elucidated the potential tumor suppressive role of Klotho in the development of T-cell lymphoma. It may serve as a potent candidate for the target therapy of T-cell lymphoma.

\section{Materials and methods}

Patients and samples. The paraffin-embedded lymph node samples were collected from 35 newly diagnosed cases of T-cell lymphoma (21 males and 14 females; age range, 19-81 years; median age, 53 years) and 20 normal lymph nodes. Histological diagnoses were established according to the WHO classification (15). Normal peripheral blood mono- 
nuclear cells (PBMCs) from healthy volunteers were isolated by Ficoll centrifugation (TBD Science, Tianjin, China). T cells were isolated with Nylon Wool Fiber Columns. This study was approved by the Medical Ethics Committee of Shandong Provincial Hospital Affiliated to Shandong University. All samples were obtained with informed consent in accordance with the Declaration of Helsinki.

Cell lines. Jurkat and Molt-3 (T-cell acute lymphocytic leukemia cell lines) were available from Typical Culture Preservation Commission Cell Bank (Chinese Academic of Science, Shanghai, China). MyLa 3676 (cutaneous T-cell lymphoma, lymphoblast, Sezary syndrome) was retained by our laboratory. Karpas 299 (ALK ${ }^{+}$ALCL cell line) was obtained from Shanghai Bioleaf Biotech Co., Ltd. All the above cell lines were maintained in PRMI-1640 (Gibco, Life Technologies, Rockville, MD, USA) containing 10\% fetal bovine serum (FBS; Gibco, Life Technologies) and $1 \%$ penicillin/streptomycin mixture.

Cell transfection. Lentivirus vectors either encoding Klotho (LV-KL) or an empty lentiviral vector (LV-Con) were generated by GeneChem (Shanghai, China). Lentivirus transfection of T-cell lymphoma cells were performed according to the manufacturer's instructions. Infection efficiencies were assessed by green fluorescent protein (GFP) fluorescence through flow cytometry. The stably transfected cells were selected with $5 \mu \mathrm{g} / \mathrm{ml}$ puromycin (Sigma-Aldrich, USA).

Immunohistochemistry (IHC). IHC was carried out with primary rabbit anti-Klotho (Abcam, Cambridge, USA). $4-\mu \mathrm{M}$-paraffin sections were deparaffinized and hydrated. Antigen retrieval was performed in $0.01 \mathrm{M}$ sodium citrate (pH 6.0) buffer under high-pressure. Endogenous peroxidase was blocked with $3 \% \mathrm{H}_{2} \mathrm{O}_{2}$ for $15 \mathrm{~min}$, followed by incubation overnight at $4^{\circ} \mathrm{C}$ with primary anti-Klotho $(1: 100)$. Then the tissue sections were treated with the second antibody from SP reagent kit (Zhongshan Golden Bridge Biotechnology Co., Ltd., Beijing, China) for $30 \mathrm{~min}$ at room temperature. Bound antibody was detected by secondary antibody and diaminobenzidine (DAB) kit (Zhongshan Golden Bridge Biotechnology). The stained slices were counterstained with hematoxylin and mounted. Negative control was performed with the primary antibody replaced by PBS. IHC staining was scored by two independent observers who were blinded to the patient clinical data. Cases with $\geq 10 \%$ positive cells were considered as positive.

Real-time quantitative polymerase chain reaction (qRT-PCR). For the qRT-PCR, total RNA was extracted with RNAiso Plus (Takara, Dalian, China). The mRNA level was detected by Nanodrop 2000 (Thermo Fisher Scientific, Waltham, MA, USA). Reverse transcription reaction was performed with reverse transcription reagents (Takara). Expression level of mRNA was quantified using SYBR Green Premix Ex Taq II kit (Takara) in LightCycler 480 real-time quantitative PCR system (Roche, Basel, Swizerland). Primers for qRT-PCR are listed in Table I. Glyceraldehyde-3-phosphate dehydrogenase $(\mathrm{GAPDH})$ was used as an internal control and relative quantification was determined by the $2^{-\Delta \Delta C t}$ method.
Table I. Primer sequences for qRT-PCR.

Gene Primer sequence

\begin{tabular}{ll} 
Klotho & F, 5'-AGCAATCTGGTCTGAATAACACTGG-3' \\
& R, 5'-CATGTTTCAGCGTGAAAGTTCAAAG-3' \\
GAPDH & F, 5'-GGGAAACTGTGGCGTGAT-3' \\
& R, 5'-GAGTGGGTGTCGCTGTTGA-3' \\
\hline
\end{tabular}

Western blotting. For western blot analysis, cell lysates were extracted by radio-immunoprecipitation assay (RIPA) buffer (Shenergy Biocolor, Shanghai, China) together with phosphatase inhibitor cocktail (PhosSTOP; Roche, Mannheim, Germany). The BCA assay (Shenergy Biocolor) was performed to detect protein concentration. Proteins $(30 \mu \mathrm{g})$ were electrophoresed on SDS-PAGE and transferred to PVDF membranes (Millipore, Billerica, MA, USA). Membranes were blocked with 5\% skim milk in Tris-buffered saline. After incubation with primary antibodies, membranes were incubated with HRP-conjugated secondary antibodies (Zhongshan Golden Bridge). Proteins were visualized by electro-chemiluminescence detection reagents (Amersham Imager; General Electric, USA). The following antibodies used for western blotting were purchased from Cell Signaling Technology: p-IGF-1R, t-IGF-1R, p-AKT, t -AKT, p-ERK1/2, t-ERK1/2, Mcl-1 and caspase-3. Klotho antibody was purchased from Abcam. The experiments were performed in triplicate with GAPDH (Zhongshan Golden Bridge) as endogenous control.

CCK-8 proliferation assay. The influence of Klotho on viability of T-cell lymphoma cells were assessed by Cell Counting Kit-8 (CCK-8; Dojindo, Kumamoto, Japan). Briefly, T-cell lymphoma cell lines stably transfected with LV-KL or LV-Con were seeded into 96 -well plates $(5,000$ cells/100 $\mu \mathrm{l} /$ well, respectively) with PRMI-1640 medium supplemented with $10 \%$ FBS. Then the cells were incubated with $10 \mu \mathrm{l} /$ well of CCK-8 and cell viability was detected by light absorption at $450 \mathrm{~nm}$ by Multiskan GO Microplate Reader (Thermo Scientific, Rockford, IL, USA).

Cell apoptosis assay. Apoptosis of T-cell lymphoma cells were tested by Annexin V-PE/7-aminoactinomycin (7AAD) kit (BD Biosciences, Bedford, MA, USA) according to the manufacturer's instructions. Cells with designed treatment were harvested and washed twice in clod PBS and resuspended in $1 \mathrm{X}$ binding buffer at a concentration of $1 \times 10^{6} \mathrm{cells} / \mathrm{ml}$, then $100 \mu \mathrm{l}$ was taken and incubated with $5 \mu \mathrm{l}$ Annexin V-PE and $5 \mu 17 \mathrm{AAD}$ for $15 \mathrm{~min}$ at room temperature in the dark for each tube. Afterwards, $400 \mu \mathrm{l}$ of $1 \mathrm{X}$ binding buffer was added and cell apoptotic rates were detected by Navios Flow Cytometer (Beckman Coulter, CA, USA).

Immunofluoresence. T-cell lymphoma cells with designed treatment were seeded onto glass slides by liquid thin layer cell smear (Xiaogan Aohua, Xiaogan, China). Thereafter, the T-cell lymphoma cells were fixed in 4\% PFA and permeabilized with $0.1 \%$ Triton X-100. The cells were blocked with 
A

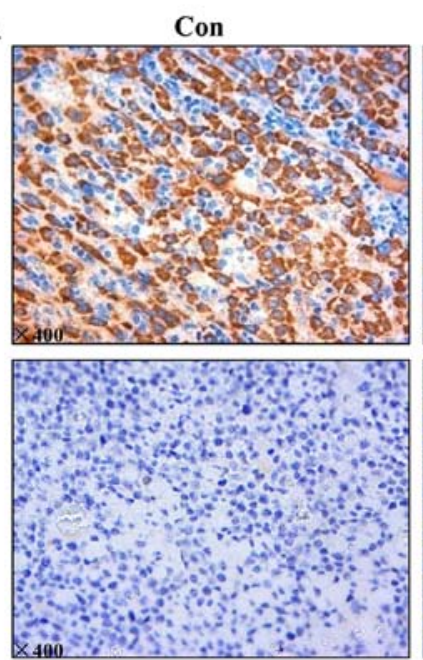

EATL
PTCL

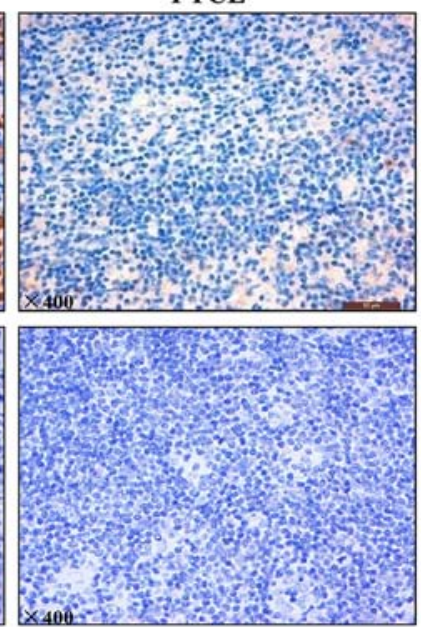

CTCL
NK/TCL

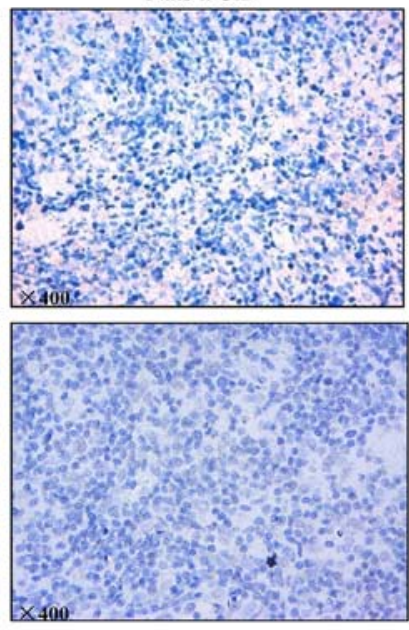

AITL
B

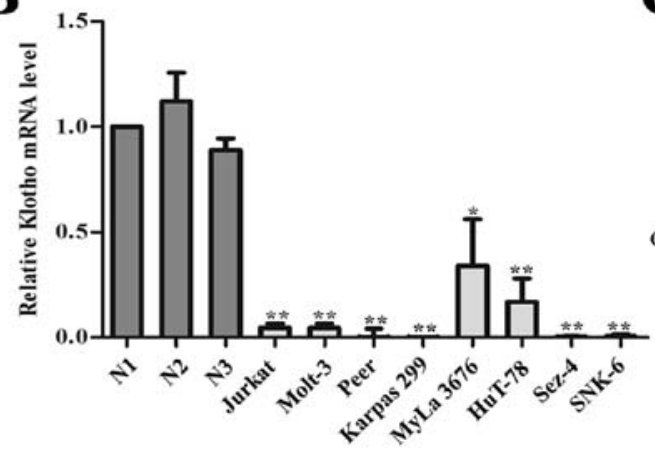

C

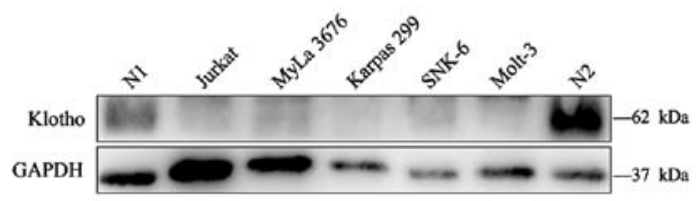

Figure 1. Expression of Klotho in the samples and cell lines of T-cell lymphoma. (A) Expression level of Klotho protein was lower in T-cell lymphoma cases than that in normal lymph nodes (original magnification, $\mathrm{x} 400$ ). (B) As detected by real-time quantitative PCR, Klotho mRNA levels were downregulated in T-cell lymphoma cell lines, compared to that in normal T cells (N1, N2 and N3). (C) Protein expression of Klotho was detected in DLBCL cells and normal T cells. ${ }^{*}<0.05,{ }^{* *} \mathrm{p}<0.01$.

Table II. Immunohistochemical expression of Klotho in T-cell lymphoma tissues.

\begin{tabular}{lccccc}
\hline Klotho expression & NK/TCL $(\mathrm{n}=12)$ & AITL $(\mathrm{n}=6)$ & CTCL $(\mathrm{n}=6)$ & EATL $(\mathrm{n}=4)$ & PTCL-NOS $(\mathrm{n}=7)$ \\
\hline Positive & $0(0 \%)$ & $1(16.7 \%)$ & $2(33.3 \%)$ & $1(25 \%)$ & $1(14.3 \%)$ \\
Negative & $12(100 \%)$ & $5(83.3 \%)$ & $4(66.7 \%)$ & $3(75 \%)$ & $6(85.7 \%)$ \\
\hline
\end{tabular}

normal goat serum for $1 \mathrm{~h}$. Then the slides were incubated with primary antibodies at $4^{\circ} \mathrm{C}$ overnight. After washing with PBS, the DyLight 488-conjugated secondary antibodies (Abbkine, CA, USA) were added. Slides were washed and mounted with 4'6-diamino-2-phenylindole (DAPI; Boster, Wuhan, China). Images were examined and recorded by Nikon IX73 fluorescent microscope.

Statistical analysis. All statistical analyses were performed by using statistic software SPSS17.0 (SPSS Inc., Chicago, IL, USA). Data are presented as the mean \pm standard deviation (mean \pm SD) from three separate experiments. Differences between groups were analyzed by one-way analysis of variance (ANOVA) or t-tests. $\mathrm{P}<0.05$ was considered statistically significant.

\section{Results}

Klotho is downregulated in T-cell lymphoma. We examined Klotho expression for primary T-cell lymphoma samples including NK/T-cell lymphoma $(n=12)$, peripheral T-cell lymphoma-not otherwise specified (PTCL-NOS, $n=7$ ), angioimmunoblasic T-cell lymphoma (AITL, $\mathrm{N}=6$ ), cutaneous T-cell lymphoma (CTCL, $n=6$ ), and enteropathy associated T-cell lymphoma (EATL, $n=4)$. Compared with normal lymph nodes, expression levels of Klotho were significantly lower in T-cell lymphoma tissues (Fig. 1A). Klotho positive rate was $14 \%$ (5 of 35 ) in T-cell lymphoma tissues whereas $75 \%$ ( 15 of 20 ) in normal lymph nodes. Cases with $\geq 10 \%$ positive cells were considered as positive. More detailed data are summarized in Table II. 

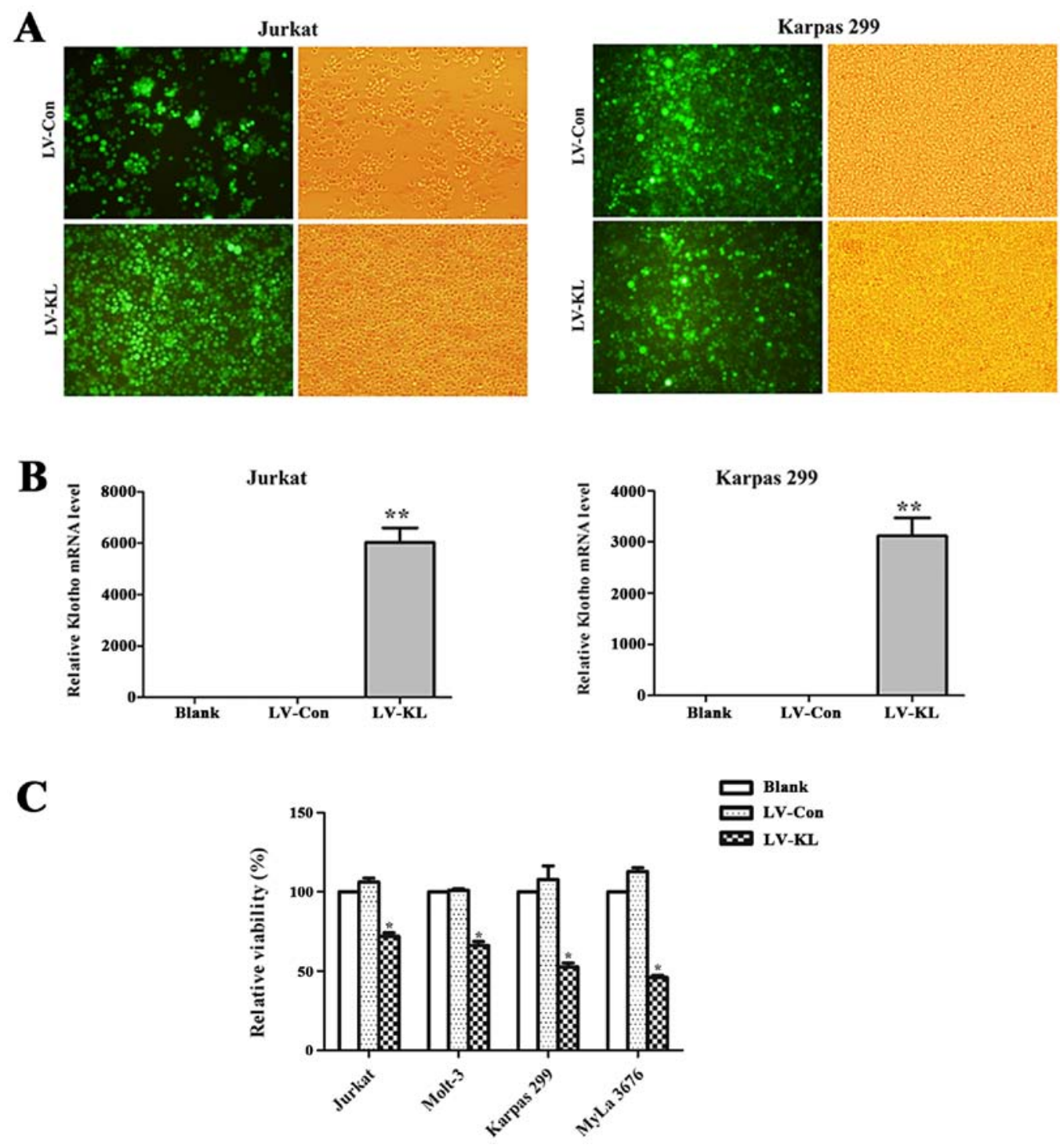

Figure 2. Klotho restrained T-cell lymphoma growth. (A) T-cell lymphoma cells were stably transfected with LV-KL. (B) Relative expression of Klotho mRNA was confirmed by quantitative PCR in T-cell lymphoma cells stably transfected with LV-KL. (C) Jurkat and Karpas 299 cells transfected with LV-KL revealed significantly lower viability compared to those transfected with LV-KL. ${ }^{*} \mathrm{p}<0.05,{ }^{* *} \mathrm{p}<0.01$.

We next confirmed the expression of Klotho in T-cell lymphoma cell lines. As shown in Fig. 1B, T-cell lymphoma cells, exhibited remarkably lower mRNA levels of Klotho compared to the peripheral blood $\mathrm{T}$ lymphocytes from healthy donors. Decreased protein levels of Klotho expression were also noted in T-cell lymphoma cell lines (Fig. 1C).

Klotho restraines T-lymphoma cell proliferation. To explore the function relevance of Klotho in the progression of T-cell lymphoma, T-cell lymphoma cell lines (Jurkat, Molt-3, Karpas 299 and MyLa 3676) were stably transfected with either Klotho-overexpression lentivirus vectors or empty vector control (Fig. 2A). Upregulated level of Klotho mRNA was confirmed by quantitative PCR (Fig. 2B). CCK-8 assay was employed to examine the viability of the above cells. Significantly decreased cell viability was noted in T-cell lymphoma cells transfected with LV-KL, compared with those transfected with LV-Con (Fig. 2C). These results indicate that Klotho inhibits the proliferation of T-cell lymphoma cells.
Klotho promotes apoptosis of T-lymphoma cells. To further investigate the mechanisms underlying the suppression of Klotho in cell proliferation by ectopic Klotho expression, Annexin V-based apoptotic assays were performed in Jurkat and Karpas 299 cell lines with stable LV-KL or LV-Con transfection. Cells transfected with LV-KL exhibited enforced apoptosis rates in T-cell lymphoma cell lines (Fig. 3A). In addition, apoptotic promotion effect of Klotho was confirmed by western blot analysis. Remarkable reduction of anti-apoptotic protein Mcl-1 and total caspase-3 protein was observed in Jurkat and Karpas 299 cell lines (Fig. 3B and C).

Klotho suppresses the activation of IGF-1R signaling in T-cell lymphoma. Having elucidated that Klotho could interfere with the viability and apoptosis of T-cell lymphoma cell lines, we next explored the involved molecular mechanisms responsible for the function of Klotho. The IGF-1R signaling plays significant role in the development of T-cell lymphoma and proliferation of T-cell lymphoma cell lines could be 
$\mathbf{A}$

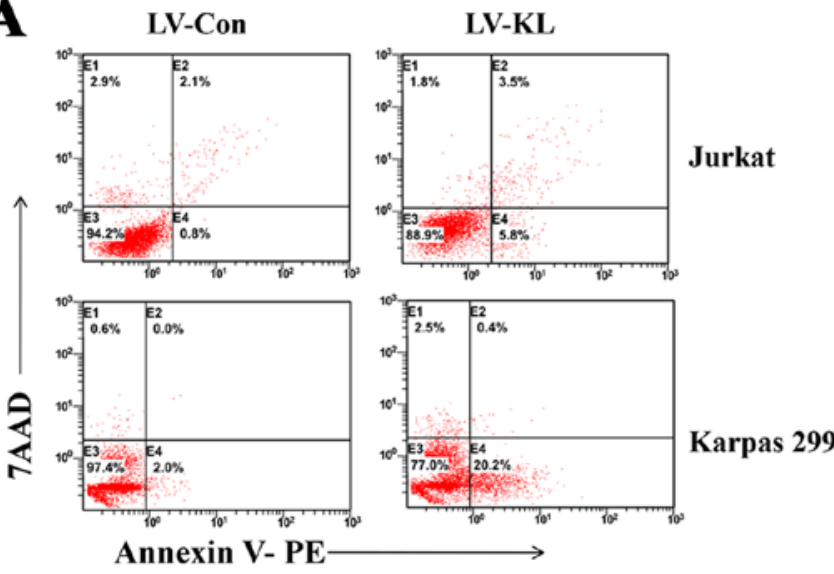

B

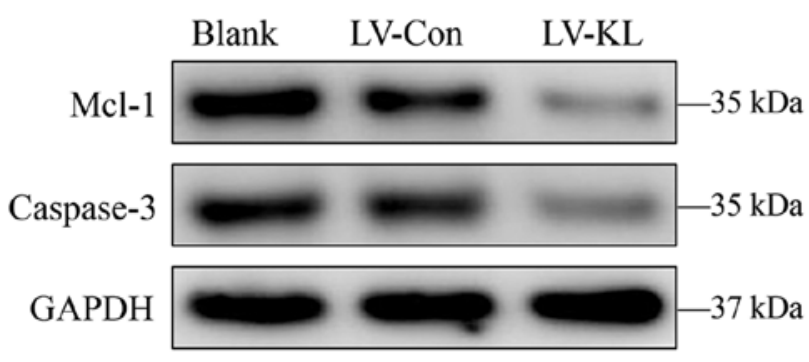

$\mathbf{C}$

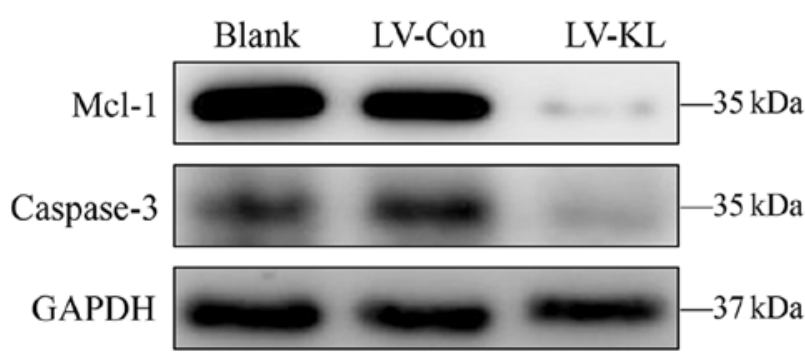

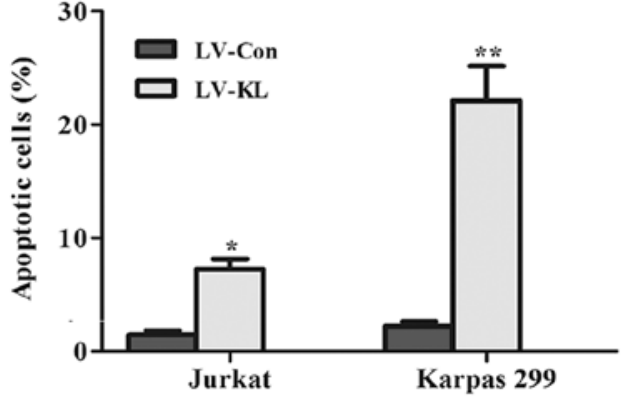

Jurkat

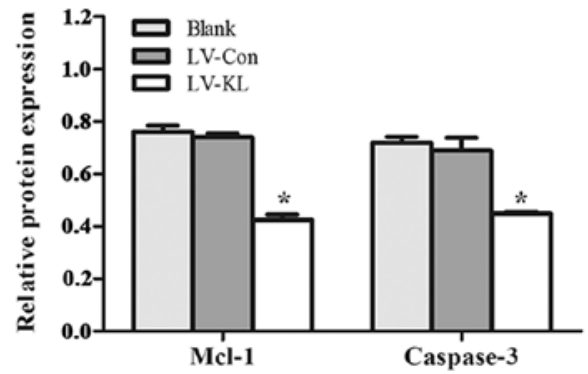

Karpas 299

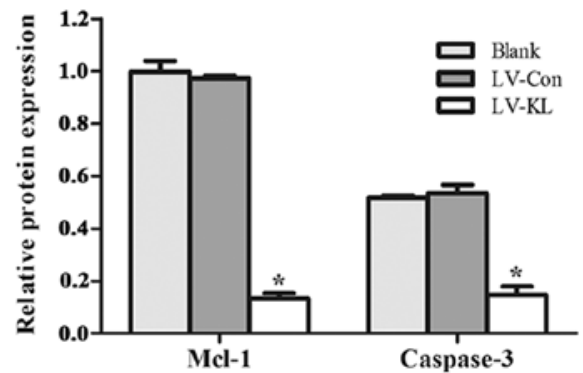

Figure 3. Klotho promotes apoptosis of T-cell lymphoma cells. (A) Overexpression of Klotho induced apoptosis of Jurkat and Karpas 299 cells. (B and C) Decreased expression of Mcl-1 and caspase- 3 were detected in T-cell lymphoma cells stably transfected with LV-KL. ${ }^{\mathrm{p}} \mathrm{p}<0.05,{ }^{* * *} \mathrm{p}<0.01$.

induced by IGF-1 exploration. CCK-8 assay was performed to determine the effect of Klotho overexpression on IGF-1induced cell proliferation. T-cell lymphoma cell lines stably transfected with either Klotho overexpressing vector or empty control vector were incubated with IGF-1 or vehicle control in $0.5 \% \mathrm{FBS}$ culture medium for $48 \mathrm{~h}$. In the group without IGF-1 treatment, LV-KL transfection induced declined viability of T-cell lymphoma cell lines compared to the empty-vector group. Furthermore, we noted that the Klotho-induced inhibition of T-cell viability was less reversed by IGF-1 treatment. With IGF-1 addition, cell proliferation of LV-Con treated cells increased by up to $50 \%$, whereas the only up to $30 \%$ enhancement of cell proliferation was found in cells with Klotho overexpression (Fig. 4A).

We next investigated the modulation effect of Klotho on IGF-1R signaling in T-cell lymphoma cell lines. Jurkat and Karpas 299 cells transfected with LV-KL revealed significantly decreased phosphorylation level of IGF-1R. Moreover, the downstream targets of IGF-1R signaling, including AKT and ERK1/2, were also inhibited by Klotho overexpression (Fig. 4B and C).

\section{Discussion}

In the present study, we provide the first evidence that Klotho as a potential tumor suppressor in the tumorigenesis of T-cell lymphoma. The decline of Klotho initiates anti-proliferative and pro-apoptotic effect through inhibiting IGF-1R signaling activation. This study illuminates the tumor suppressive effect of Klotho and highlights the potential application of Klotho in the targeted therapy in T-cell lymphoma.

The tumor suppressive function of Klotho has been reported in several human solid malignancies, but less in hematological cancers, especially in lymphoma (16-19). Lower Klotho expression was detected in T-cell lymphoma tissues whereas high expression level of Klotho was observed in normal lymph 

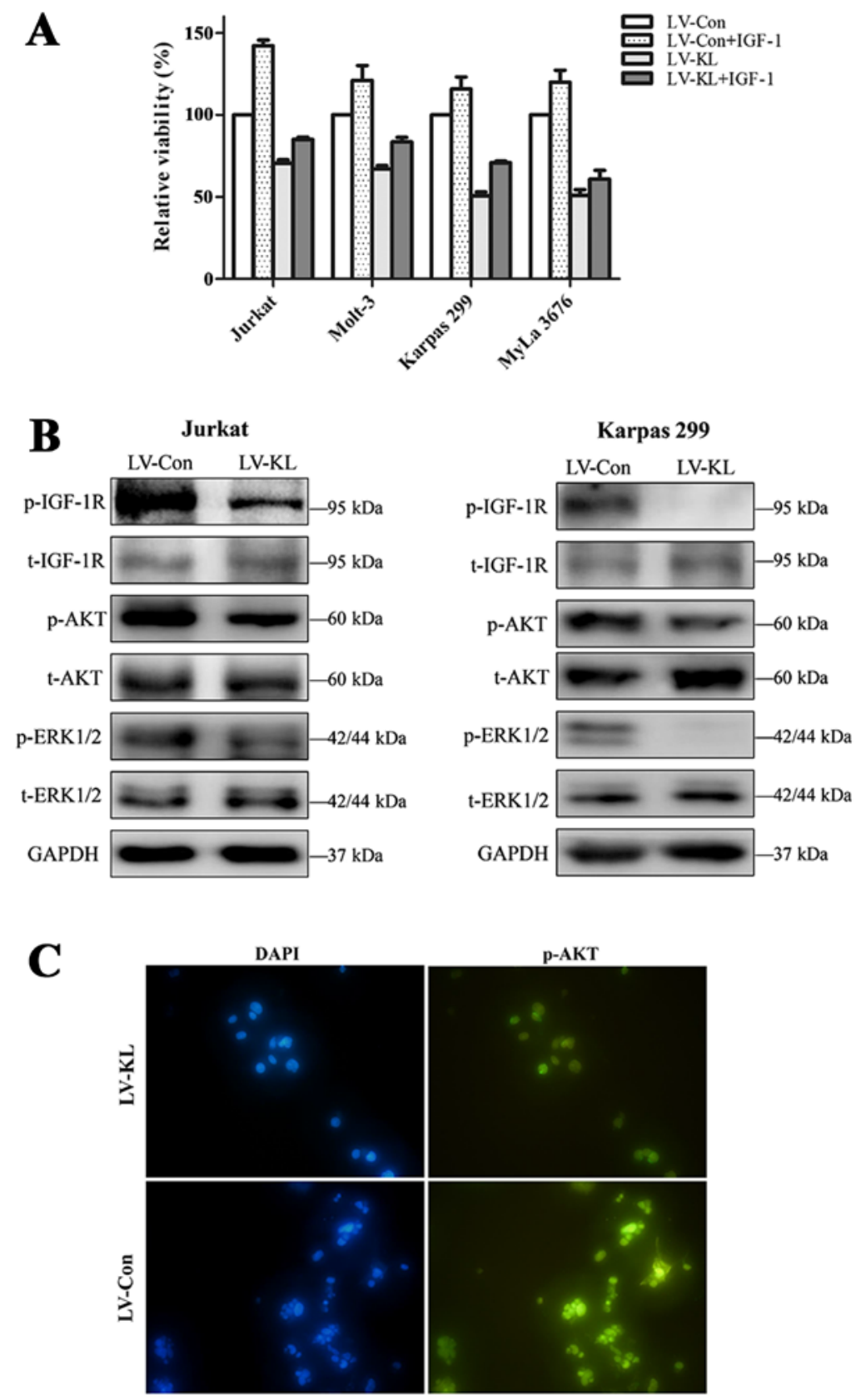

Figure 4. Klotho inhibits the IGF-1R signaling in T-cell lymphoma. (A) IGF-1 induced proliferation of T-cell lymphoma cells was inhibited by Klotho overexpression. Jurkat, Molt-3, Karpas 299 and MyLa 3676 were stably transfected with LV-KL or LV-Con, serum starved for $48 \mathrm{~h}$ and treated with IGF-1 followed by CCK-8 analysis. (B) Jurkat and Karpas 299 cells stably expressing LV-KL presented decreased phosphorylation levels of IGF-1R, Akt and ERK1/2. (C) Representative images of immunofluorescence analysis of AKT in Jurkat cells with Klotho overexpression. Magnification, x400.

nodes, in accordance with recent studies showing decreased Klotho expression in human malignancies. Recently, accumulating evidence demonstrated several mechanisms involved in the aberrant expression of Klotho in solid cancers, including epigenetic mechanisms and autophagy $(17,20,21)$. Additionally, Klotho decline has a significant prognostic value in cancers, such as large cell neuroendocrine carcinoma (LCNEC), small cell lung cancer (SCLC) and hepatocellular carcinoma (22-24). Usuda et al (22) reported that Klotho staining provided a novel biomarker for prognosis in patients with LCNEC, especially for those without lymphangioinvasion or lymph node metastasis. It suggested that evaluation of Klotho in cancer may improve the personalized treatment in human malignancies. T-cell lymphoma is characterized by heterogeneity in clinical, molecular and biological presentations. The clinical diagnosis of T-cell lymphoma is primary based on the biopsy histopathology (25). With the deepening of further investigations, lower Klotho expression may serve as a potential marker for the pathological diagnosis of T-cell lymphoma.

Furthermore, our data elucidated that the cell growth of T-cell lymphoma could be restrained by Klotho. Overexpression of Klotho inhibited the proliferation and induced apoptosis of T-cell lymphoma cells. The results are consistent with the studies in lung cancer $(7,17)$. Moreover, it has been reported that soluble Klotho and conditioned medium from Klotho- 
overexpressing cells could suppress the growth of pancreatic cancer and breast cancer cells $(16,26)$. However, investigations with soluble Klotho and in vivo studies are still needed to be conducted to explore the effective domain of Klotho protein and evaluate the safety and efficacy, and pharmacokinectic mechanisms of Klotho in T-cell lymphoma.

Interestingly, we observed the suppressive effect of Klotho on the activation of IGF-1R signaling in T-cell lymphoma. Our findings are consistent with recent reports which elucidated that Klotho could serve as a regulator of IGF-1R signaling in cancer. Aberrant activation of IGF-1R signaling was involved in the development and progression of T-cell lymphoma $(14,27)$. Moreover, structure-function analysis indicated that Klotho could interact with the IGF-1R (28). Klotho-induced suppression of IGF-1R pathway may acts as a novel mechanism involved in the pathogenesis of T-cell lymphoma. Additionally, several signaling pathways, such as Wnt signaling, FGF23 signaling and PI3K signaling, are also reported to participate in the biological mechanism of Klotho $(16,29,30)$. Further investigations are still needed to clarify the detailed mechanism involved in the development different types of tumors.

In conclusion, this study identified that Klotho acted as a novel tumor suppressor in T-cell lymphoma. We elucidated the crucial role of Klotho in inhibiting tumor cell proliferation and inducing cell apoptosis in T-cell lymphoma. The tumor suppressive effect of Klotho may be mediated by inhibiting the activation IGF-1R signaling pathway. Being an endogenous circulating hormone, the secreted Klotho could function as an active form and inhibit the tumor growth safely and effectively in mice. These data suggested that Klotho may serve as a hopeful target for the development of novel therapeutic strategy of T-cell lymphoma.

\section{Acknowledgements}

This study was supported by the National Natural Science Foundation (nos. 81473486 and 81270598), National Public Health Grand Research Foundation (no. 201202017), Natural ScienceFoundations of ShandongProvince(nos.ZR2012HZ003 and 2009ZRB14176), Technology Development Projects of Shandong Province (nos. 2014GSF118021, 2010GSF10250 and 2008GG2NS02018), Program of Shandong Medical Leading Talent, and Taishan Scholar Foundation of Shandong Province.

\section{References}

1. Zhao WL: Targeted therapy in T-cell malignancies: Dysregulation of the cellular signaling pathways. Leukemia 24: 13-21, 2010.

2. Kuro-o M, Matsumura Y, Aizawa H, Kawaguchi H, Suga T, Utsugi T, Ohyama Y, Kurabayashi M, Kaname T, Kume E, et al: Mutation of the mouse klotho gene leads to a syndrome resembling ageing. Nature 390: 45-51, 1997.

3. Kurosu H, Yamamoto M, Clark JD, Pastor JV, Nandi A, Gurnani P, McGuinness OP, Chikuda H, Yamaguchi M, Kawaguchi H, et al: Suppression of aging in mice by the hormone Klotho. Science 309: 1829-1833, 2005

4. Kuro-o M: Klotho and aging. Biochim Biophys Acta 1790: 1049-1058, 2009.

5. Yamamoto M, Clark JD, Pastor JV, Gurnani P, Nandi A, Kurosu H, Miyoshi M, Ogawa Y, Castrillon DH, Rosenblatt KP, et al: Regulation of oxidative stress by the anti-aging hormone klotho. J Biol Chem 280: 38029-38034, 2005.

6. Zhou X and Wang X: Klotho: A novel biomarker for cancer. J Cancer Res Clin Oncol 141: 961-969, 2015.
7. Dai D, Wang Q, Li X, Liu J, Ma X and Xu W: Klotho inhibits human follicular thyroid cancer cell growth and promotes apoptosis through regulation of the expression of stanniocalcin-1. Oncol Rep 35: 552-558, 2016

8. Rubinek T, Shulman M, Israeli S, Bose S, Avraham A Zundelevich A, Evron E, Gal-Yam EN, Kaufman B and Wolf I: Epigenetic silencing of the tumor suppressor klotho in human breast cancer. Breast Cancer Res Treat 133: 649-657, 2012.

9. Chang B, Kim J, Jeong D, Jeong Y, Jeon S, Jung SI, Yang Y, Kim KI, Lim JS, Kim C, et al: Klotho inhibits the capacity of cell migration and invasion in cervical cancer. Oncol Rep 28: 1022-1028, 2012.

10. Yaktapour N, Ubelhart R, Schuler J, Aumann K, Dierks C, Burger M, Pfeifer D, Jumaa H, Veelken H, Brummer T, et al: Insulin-like growth factor-1 receptor (IGF1R) as a novel target in chronic lymphocytic leukemia. Blood 122: 1621-1633, 2013.

11. Vishwamitra D, Shi P, Wilson D, Manshouri R, Vega F, Schlette EJ and Amin HM: Expression and effects of inhibition of type I in sulin-like growth factor receptor tyrosine kinase in mantle cell lymphoma. Haematologica 96: 871-880, 2011.

12. Mathur R, Sehgal L, Braun FK, Berkova Z, Romaguerra J, Wang M, Rodriguez MA, Fayad L, Neelapu SS and Samaniego F: Targeting Wnt pathway in mantle cell lymphoma-initiating cells. J Hematol Oncol 8: 63, 2015.

13. Groen RW, Oud ME, Schilder-Tol EJ, Overdijk MB, ten Berge D, Nusse R, Spaargaren M and Pals ST: Illegitimate WNT pathway activation by beta-catenin mutation or autocrine stimulation in T-cell malignancies. Cancer Res 68: 6969-6977, 2008.

14. Huang Z, Fang Z, Zhen H, Zhou L, Amin HM and Shi P: Inhibition of type I insulin-like growth factor receptor tyrosine kinase by picropodophyllin induces apoptosis and cell cycle arrest in T lymphoblastic leukemia/lymphoma. Leuk Lymphoma 55: 1876-1883, 2014.

15. Jaffe ES: The 2008 WHO classification of lymphomas: Implications for clinical practice and translational research. Hematology (Am Soc Hematol Educ Program) 2009: 523-531, 2009.

16. Abramovitz L, Rubinek T, Ligumsky H, Bose S, Barshack I, Avivi C, Kaufman B and Wolf I: KL1 internal repeat mediates klotho tumor suppressor activities and inhibits bFGF and IGF-I signaling in pancreatic cancer. Clin Cancer Res 17: 4254-4266, 2011.

17. Chen B, Ma X, Liu S, Zhao W and Wu J: Inhibition of lung cancer cells growth, motility and induction of apoptosis by Klotho, a novel secreted Wnt antagonist, in a dose-dependent manner. Cancer Biol Ther 13: 1221-1228, 2012.

18. Li XX, Huang LY, Peng JJ, Liang L, Shi DB, Zheng HT and Cai SJ: Klotho suppresses growth and invasion of colon cancer cells through inhibition of IGF1R-mediated PI3K/AKT pathway. Int J Oncol 45: 611-618, 2014.

19. Aviel-Ronen S, Rubinek T, Zadok O, Vituri A, Avivi C, Wolf I and Barshack I: Klotho expression in cervical cancer: Differential expression in adenocarcinoma and squamous cell carcinoma. J Clin Pathol 69: 53-57, 2016.

20. Lee J, Jeong DJ, Kim J, Lee S, Park JH, Chang B, Jung SI, Yi L, Han Y, Yang Y, et al: The anti-aging gene Klotho is a novel target for epigenetic silencing in human cervical carcinoma. Mol Cancer 9: 109, 2010.

21. Chen T, Ren H, Thakur A, Yang T, Li Y, Zhang S, Wang T and Chen M: Decreased level of klotho contributes to drug resistance in lung cancer cells: Involving in klotho-mediated cell autophagy. DNA Cell Biol 35: 751-757, 2016.

22. Usuda J, Ichinose S, Ishizumi T, Ohtani K, Inoue T, Saji H, Kakihana M, Kajiwara N, Uchida O, Nomura M, et al: Klotho is a novel biomarker for good survival in resected large cell neuroendocrine carcinoma of the lung. Lung Cancer 72: 355-359, 2011.

23. Xie B, Zhou J, Yuan L, Ren F, Liu DC, Li Q and Shu G: Epigenetic silencing of Klotho expression correlates with poor prognosis of human hepatocellular carcinoma. Hum Pathol 44: 795-801, 2013.

24. Usuda J, Ichinose S, Ishizumi T, Ohtani K, Inoue T, Saji H, Kakihana M, Kajiwara N, Uchida O, Nomura M, et al: Klotho predicts good clinical outcome in patients with limited-disease small cell lung cancer who received surgery. Lung Cancer 74: 332-337, 2011.

25. Tilly H, Gomes da Silva M, Vitolo U, Jack A, Meignan M, Lopez-Guillermo A, Walewski J, André M, Johnson PW, Pfreundschuh M, et al; ESMO Guidelines Committee: Diffuse large B-cell lymphoma (DLBCL): ESMO Clinical Practice Guidelines for diagnosis, treatment and follow-up. Ann Oncol 26 (Suppl 5): v116-v125, 2015. 
26. Wolf I, Levanon-Cohen S, Bose S, Ligumsky H, Sredni B, Kanety H, Kuro-o M, Karlan B, Kaufman B, Koeffler HP, et al: Klotho: A tumor suppressor and a modulator of the IGF-1 and FGF pathways in human breast cancer. Oncogene 27: 7094-7105, 2008 .

27. Vishwamitra D, Curry CV, Alkan S, Song YH, Gallick GE, Kaseb AO, Shi P and Amin HM: The transcription factors Ik-1 and MZF1 downregulate IGF-IR expression in NPM-ALK(+) T-cell lymphoma. Mol Cancer 14: 53, 2015.

28. Ligumsky H, Rubinek T, Merenbakh-Lamin K, Yeheskel A, Sertchook R, Shahmoon S, Aviel-Ronen S and Wolf I: Tumor suppressor activity of klotho in breast cancer is revealed by structure-function analysis. Mol Cancer Res 13: 1398-1407, 2015.
29. Sun H, Gao Y, Lu K, Zhao G, Li X, Li Z and Chang H: Overexpression of Klotho suppresses liver cancer progression and induces cell apoptosis by negatively regulating wnt $/ \beta$-catenin signaling pathway. World J Surg Oncol 13: 307, 2015.

30. Zhu Y, Xu L, Zhang J, Xu W, Liu Y, Yin H, Lv T, An H, Liu L, $\mathrm{He} \mathrm{H}$, et al: Klotho suppresses tumor progression via inhibiting PI3K/Akt/GSK3//Snail signaling in renal cell carcinoma. Cancer Sci 104: 663-671, 2013. 